J. Nonlinear Var. Anal. 4 (2020), No. 3, pp. 455-468

Available online at http://jnva.biemdas.com

https://doi.org/10.23952/jnva.4.2020.3.09

\title{
TWO SOLUTIONS FOR A PARAMETRIC SINGULAR $p$-LAPLACIAN PROBLEM
}

\author{
PASQUALE CANDITO $^{1, *}$, UMBERTO GUARNOTTA $^{2}$, KANISHKA PERERA $^{3}$ \\ ${ }^{1}$ Department DICEAM, University of Reggio Calabria, via Graziella (Feo Di Vito), 89122 Reggio Calabria, Italy \\ ${ }^{2}$ Dipartimento di Matematica e Informatica, Università di Catania, Viale A. Doria 6, 95125 Catania, Italy \\ ${ }^{3}$ Department of Mathematical Sciences, Florida Institute of Technology, Melbourne, FL 32901, USA
}

Abstract. In this paper, we combine variational methods and truncation techniques to study the existence of at least two weak solutions for a $p$-Laplacian problem depending by a positive parameter $\lambda$ and with reaction term singular at zero. A quantitative estimate of the parameters is also given.

Keywords. Quasilinear elliptic equations; Singular term; Variational methods; $p$-Laplacian problem.

\section{INTRODUCTION}

Starting form the pioneering papers [14] and [31], singular elliptic equations have been extensively studied by many scholars, so much so that it is impossible to summarize the many and important contributions present in the literature on this topic. Some of those ones, closely related to what are studied in this paper, can be found, for instance, in [13, 15], and the monograph [18], dealing with semilinear problems. For $p$-Laplace equations, we refer to $[19,20,21,22,26,28,29]$ and the references therein.

Let $N \geq 3$ and $\Omega \subseteq \mathbb{R}^{N}$ be a bounded domain with $C^{2}$-boundary $\partial \Omega$. In this paper, we study the existence of at least two weak solutions for the following Dirichlet problem:

$$
\begin{cases}-\Delta_{p} u=\lambda f(x, u) & \text { in } \Omega, \\ u>0 & \text { in } \Omega, \\ u=0 & \text { on } \partial \Omega,\end{cases}
$$

where $1<p<N, \Delta_{p} u:=\operatorname{div}\left(|\nabla u|^{p-2} \nabla u\right)$ denotes the $p$-Laplacian operator, $\lambda>0$ is a parameter, and $f: \Omega \times(0,+\infty) \rightarrow[0,+\infty)$ is a Carathéodory function satisfying the following assumptions:

$\underline{\mathrm{H}(\mathrm{f})_{1}}: \lim _{s \rightarrow 0^{+}} f(x, s)=+\infty$, uniformly w.r.t. $x \in \Omega$.

$\mathrm{H}(\mathrm{f})_{2}$ : There exist $s_{0}, b_{1}>0, b_{2} \geq 0, \gamma \in(0,1), q \in\left(1, p^{*}\right)$, and $a \in L^{q^{\prime}}(\Omega)_{+}$such that:

(i) $f(x, s) s^{\gamma} \leq b_{1}$, for a.a. $x \in \Omega$ and for all $s \in\left(0, s_{0}\right)$;

(ii) $f(x, s) \leq a(x)+b_{2} s^{q-1}$ for a.a. $x \in \Omega$ and for all $s \in\left[s_{0},+\infty\right)$, with $p^{*}:=\frac{N p}{N-p}$.

*Corresponding author.

E-mail addresses: pasquale.candito@unirc.it (P. Candito), umberto.guarnotta@phd.unict.it (U. Guarnotta), kperera@ fit.edu (K. Perera).

Received July 10, 2020; Accepted August 16, 2020.

(C)2020 Journal of Nonlinear and Variational Analysis 
Moreover, in order to obtain a second solution in the superlinear case, that is, $p<q<p^{*}$, we also require that $f$ fulfills the Ambrosetti-Rabinowitz condition (unilater version; cf. [26, pag. 154] and also [28, 29]):

$\left(\mathrm{AR}^{+}\right):$There exist $\mu>p, R>s_{0}$, and $s_{1} \in\left[s_{0}, R\right)$ such that

$$
0<\inf _{x \in \Omega} \int_{0}^{R} f(x, t) d t, \quad 0<\mu \int_{s_{1}}^{s} f(x, t) d t \leq f(x, s) s,
$$

for a.a. $x \in \Omega$ and for all $s \geq R$, where $s_{0}$ is as in $\mathrm{H}(\mathrm{f})_{2}$.

Roughly speaking, we ensure the existence of at least two weak solutions for problem $\left(P_{\lambda, f}\right)$ by looking for a local minimizer and a mountain pass critical point for a suitable energy functional $J_{\lambda}$ associated to it (Theorem 3.2).

A crucial point in the analysis of both regular and singular problems via variational methods is to guarantee that a suitable energy functional satisfies the classical mountain pass geometry [2]. The truncation methods based on the so-called $W^{1, p}$ versus $C^{1}$ local minimizers results, introduced in [4], and the regularity theory for PDEs (cf. [9, 17, 20, 21]) are powerful tools to the purpose. See also the more recent bifurcation results collected in [26].

In this paper, motivated by $[8,10,11,28,29]$, we get the first solution for $\left(P_{\lambda, f}\right)$ as a local minimizer by applying a new version (Theorem 2.1) of a critical point result due to [7] (see also [6]). In particular, unlike [28, 29], we do not adopt perturbation arguments to get a local minimum. In view of $[8,10,11]$, we achieve the goal by introducing a suitable truncation $f^{*}$, only from below, of the reaction term $f$. In order to preserve the $C^{1}$-regularity of $J_{\lambda}$, we extend $f^{*}$ for $s<0$ by symmetry (Lemmas 2.1 and 2.2). Although that new variational framework forces us to assume the function $f$ to be positive, it allows us to get some advantages as, for instance,

- a quantitative estimate of the parameter $\lambda$ is provided thanks to [32];

- the nonlinear term $f(x, u)$ may be non-monotone with respect to $u$, and therefore we can apply our results for a wider class of functions than those ones usually considered in the aforementioned papers, where $f$ appears as the polynomial perturbation of the pure singular case, i.e., $f(x, u)=a(x) u^{-\gamma}+\mu g(x, u)$, where $a$ and $g$ are suitable functions, and $\mu \in \mathbb{R}, 0<\gamma<1$ are opportune constants.

Section 2 is devoted to some preliminary results, while the main results (Theorems 3.1 and 3.2) are contained in Section 3.

\section{Preliminaries}

Let $N \geq 3$ and $\Omega \subseteq \mathbb{R}^{N}$ be a bounded domain with $C^{2}$-boundary $\partial \Omega$.

Let $Y$ be a real-valued function space, and $u, v \in Y$. We say that $u \leq v$ if $u(x) \leq v(x)$ for a.a. $x$. Moreover, we define $Y_{+}:=\{u \in Y: u \geq 0\}$. Hereafter, we denote by $d$ the 'distance function':

$$
d: \Omega \rightarrow[0,+\infty), \quad d(x):=\operatorname{dist}(x, \partial \Omega)=\min _{y \in \partial \Omega}|x-y|,
$$

where $|\cdot|$ is the standard $N$-dimensional Euclidean norm. Symbol $\|\cdot\|_{p}$ denotes the norm in the Lebesgue space $L^{p}(\Omega)$, i.e.,

$$
\|u\|_{p}:=\left(\int_{\Omega}|u(x)|^{p} d x\right)^{\frac{1}{p}} \quad \forall u \in L^{p}(\Omega),
$$


while $\|\cdot\|$ is the usual equivalent norm in $W_{0}^{1, p}(\Omega)$, i.e., $\|u\|:=\|\nabla u\|_{p}$ for any $u \in W_{0}^{1, p}(\Omega)$. Moreover, one has that the embedding $W_{0}^{1, p}(\Omega) \hookrightarrow L^{q}(\Omega)$ is compact for every $q \in\left[1, p^{*}\right)$, where $p^{*}:=\frac{N p}{N-p}$ is the critical Sobolev exponent, as usual. Here, $C_{q}$ stands for the best constant in the embedding inequality

$$
\|u\|_{q} \leq C_{q}\|u\| \quad \forall u \in W_{0}^{1, p}(\Omega) .
$$

Taking into account [32], we can obtain that

$$
C_{q} \leq S_{q}:=\pi^{-1 / 2} N^{-1 / p}|\Omega|^{\frac{p^{*}-q}{p^{*} q}}\left(\frac{p-1}{N-p}\right)^{1-1 / p}\left\{\frac{\Gamma(1+N / 2) \Gamma(N)}{\Gamma(N / p) \Gamma(1+N-N / p)}\right\},
$$

where $\Gamma$ stands for the Gamma function.

Moreover, we denote by $\lambda_{1}$ and $\varphi_{p}$ the first eigenvalue and the corresponding ( $L^{p}$-normalized) positive eigenfunction associated to the negative $p$-Laplacian in $W_{0}^{1, p}(\Omega)$. In other words, $\varphi_{p}$ solves problem $\left(P_{\lambda_{1},|u|^{p-2} u}\right)$. The strong maximum principle [30] and the regularity theory [24] ensure that

$$
\varphi_{p} \in C_{0}^{1, \alpha}(\bar{\Omega}), 0<\alpha \leq 1 \quad \text { and } \quad \tilde{l} d(x) \leq \varphi_{p}(x) \leq \tilde{L} d(x),
$$

for all $x \in \bar{\Omega}$, where $\tilde{l}$ and $\tilde{L}$ are two positive constants, and $d$ is as in (2.1).

Owing to [23], (2.3) implies that $d(x)^{-\gamma} \in L^{1}(\Omega)$, and the following classical inequality holds (cf. [1]).

Proposition 2.1 (Hardy-Sobolev's inequality). Let $p \in(1, N), \tau \in[0,1]$ and set $\frac{1}{r}=\frac{1}{p}-\frac{1-\tau}{N}$. Then, for any $u \in W_{0}^{1, p}(\Omega)$, one has $u d^{-\tau} \in L^{r}(\Omega)$ with

$$
\left\|u d^{-\tau}\right\|_{r} \leq D_{\tau}\|u\|
$$

where $D_{\tau}>0$ is a constant independent of $u$.

Furthermore (see, for instance, [12]), the regularity results and the estimate (2.3) also hold true for a weak solution $u$ of problem $\left(P_{1, g}\right)$, where $g \in L^{q}(\Omega)$ is a non-negative and non-trivial function, and $q>\frac{N p}{N p-N+p}$.

In order to fix an auxiliary variational setting, the first step is to show that problem $\left(P_{\lambda, f}\right)$ admits a subsolution.

Lemma 2.1. Let $\mathrm{H}(\mathrm{f})_{1}$ hold true and $s_{0}$ be as in $\mathrm{H}(\mathrm{f})_{2}(\mathrm{i})$. Then, for every $\lambda>0$, there exist $0<\delta<s_{0}, l=l(\lambda)>0$, and $\underline{u} \in C_{0}^{1, \alpha}(\bar{\Omega})$, where $\alpha$ is as in (2.3), such that $\underline{u}$ is a subsolution to problem $\left(P_{\lambda, f}\right)$, that is,

$$
\int_{\Omega}|\nabla \underline{u}|^{p-2} \nabla \underline{u} \nabla v d x \leq \lambda \int_{\Omega} f(x, \underline{u}) v d x \quad \forall v \in W_{0}^{1, p}(\Omega)_{+},
$$

and

$$
l d(x) \leq \underline{u}(x) \leq \delta \quad \forall x \in \bar{\Omega}
$$

where d is defined in (2.1).

Proof. Trivially, $\mathrm{H}(\mathrm{f})_{1}$ provides $\delta>0$ small enough such that

$$
f(x, s) \geq 1 \quad \forall(x, s) \in \Omega \times(0, \delta] .
$$


Let us fix any $\lambda>0$. Arguing as above (see, e.g., [27, Appendix E]), we obtain that the unique solution $e_{\lambda}$ of the problem

$$
\begin{cases}-\Delta_{p} u=\lambda & \text { in } \Omega, \\ u=0 & \text { on } \partial \Omega,\end{cases}
$$

satisfies condition (2.3) (with $e_{\lambda}$ instead of $\varphi_{p}$ ). Set $M=M(\lambda):=\max \left\{\max _{\bar{\Omega}} e_{\lambda}, \delta\right\}$ and $\underline{u}:=\frac{\delta}{M} e_{\lambda}$. For every $x \in \Omega$, one has

$$
\frac{\delta}{M} \tilde{l} d(x) \leq \frac{\delta}{M} e_{\lambda}(x)=\underline{u}(x) \leq \frac{\delta}{M} \max _{\bar{\Omega}} e_{\lambda} \leq \delta,
$$

so (2.6) is proved. Moreover, (2.7) yields

$$
-\Delta_{p} \underline{u}(x)=\left(\frac{\delta}{M}\right)^{p-1}\left(-\Delta_{p} e_{\lambda}(x)\right) \leq \lambda \leq \lambda f(x, \underline{u}(x)),
$$

for any $x \in \Omega$. Integration by parts concludes the proof.

To avoid unnecessary technicalities, "for all $x$ " will take the place of "for almost all $x$ ". Hereafter, we will use $c_{1}, c_{2}, \ldots$, to denote the suitable positive constants which may change their values line by line, and we will write $\underline{u}$ instead of $\underline{u}(x)$ where no confusion arises.

Take any $\underline{u}$ as in Lemma 2.1 and define the following function $f^{*}: \Omega \times \mathbb{R} \rightarrow \mathbb{R}$ as

$$
f^{*}(x, s)= \begin{cases}f(x, s), & \text { if } s \geq \underline{u} \\ f(x, \underline{u}), & \text { if }|s|<\underline{u} \\ f(x,-s), & \text { if } s \leq-\underline{u}\end{cases}
$$

It is straightforward that $f^{*}$ is a Carathéodory function. Exploiting (2.6), besides $\mathrm{H}(\mathrm{f})_{2}$, we get

$$
f^{*}(x, s) \leq \begin{cases}b_{1} \underline{u}^{-\gamma} \leq \tilde{b}_{1} d^{-\gamma}, & \text { if }|s|<s_{0} \\ a(x)+b_{2}|s|^{q-1}, & \text { if }|s| \geq s_{0}\end{cases}
$$

where $\tilde{b}_{1}=b_{1} l^{-\gamma}$. Hence, using (2.4), the Hölder inequality, and (2.2), one gets

$$
\begin{aligned}
& \left|\int_{\Omega} f^{*}(x, u) v d x\right| \leq \int_{\Omega} f^{*}(x, u)|v| d x \\
& \leq \tilde{b}_{1} \int_{\Omega}|v| d(x)^{-\gamma} d x+b_{2} \int_{\Omega}|u|^{q-1}|v| d x+\int_{\Omega} a(x)|v| d x \\
& \leq c_{1}\left(\|v\|+\|u\|_{q}^{q-1}\|v\|_{q}+\|a\|_{q^{\prime}}\|v\|_{q}\right) \\
& \leq c_{2}\left(1+\|u\|^{q-1}+\|a\|_{q^{\prime}}\right)\|v\|<+\infty
\end{aligned}
$$

for every $u, v \in W_{0}^{1, p}(\Omega)$. Therefore, problem $\left(P_{\lambda, f^{*}}\right)$ admits the equivalent weak formulation

$$
\int_{\Omega}|\nabla u|^{p-2} \nabla u \nabla v d x=\lambda \int_{\Omega} f^{*}(x, u) v d x \quad \forall v \in W_{0}^{1, p}(\Omega) .
$$

A key-point in our approach is the following

Lemma 2.2. For any $\lambda>0$, any solution to $\left(P_{\lambda, f^{*}}\right)$ is a solution of $\left(P_{\lambda, f}\right)$. 
Proof. Fix $\lambda>0$ and let $u \in W_{0}^{1, p}(\Omega)$ be a weak solution to $\left(P_{\lambda, f^{*}}\right)$. It is sufficient to prove that $u \geq \underline{u}$. First, we show that $u \geq 0$. Indeed, testing (2.12) with $v=-u^{-}$, it follows that

$$
\int_{\Omega}\left|\nabla u^{-}\right|^{p} d x=\lambda \int_{\Omega} f^{*}(x, u)\left(-u^{-}\right) d x \leq 0
$$

which implies $\left\|u^{-}\right\|=0$, that is, $u \geq 0$ in $W_{0}^{1, p}(\Omega)$.

Recall (2.5) and test (2.12) again with $v=(\underline{u}-u)^{+} \in W_{0}^{1, p}(\Omega)$, we infer

$$
\int_{\Omega}|\nabla \underline{u}|^{p-2} \nabla \underline{u} \nabla(\underline{u}-u)^{+} d x \leq \lambda \int_{\Omega} f(x, \underline{u})(\underline{u}-u)^{+} d x
$$

and

$$
\int_{\Omega}|\nabla u|^{p-2} \nabla u \nabla(\underline{u}-u)^{+} d x=\lambda \int_{\Omega} f^{*}(x, u)(\underline{u}-u)^{+} d x .
$$

Subtracting (2.13) and (2.14) term by term, we deduce from (2.9) that

$$
\int_{\Omega}\left[|\nabla \underline{u}|^{p-2} \nabla \underline{u}-|\nabla u|^{p-2} \nabla u\right](\underline{u}-u)^{+} d x \leq 0 .
$$

Hence, according to the strict monotonicity of $p$-Laplacian (cf. [25, Remark 12.13]), we conclude $\left\|(\underline{u}-u)^{+}\right\|=0$, that is, $u \geq \underline{u}$.

Set

$$
F: \Omega \times \mathbb{R} \rightarrow \mathbb{R}, \quad F(x, s)=\int_{0}^{s} f^{*}(x, t) d t
$$

and

$$
\Psi: W_{0}^{1, p}(\Omega) \rightarrow \mathbb{R}, \quad \Psi(u)=\int_{\Omega} F(x, u) d x .
$$

Clearly, $F$ is a Carathéodory function. Bearing in mind (2.10), one has

$$
f^{*}(x, s) \leq a_{1}(x)+b_{2}|s|^{q-1},
$$

for all $x \in \Omega$, where $a_{1}=c_{1} d^{-\gamma}+a \in L^{1}(\Omega)$ with $c_{1}$ being a suitable constant. Function $a_{1}$ does not satisfy the standard summability condition $a_{1} \in L^{q^{\prime}}(\Omega)$, which ensures the Nemyskii operator $N_{F} u:=F(x, u)$ associated to $F$ to be of class $C^{1}$ in $L^{q}(\Omega)$. Thus, for the reader's convenience, we collect in the following lemma some properties of the integral functional $\Psi$, and, for the sake of completeness, we also give a sketch of their proofs.

Lemma 2.3. Under hypotheses $\mathrm{H}(\mathrm{f})_{1}$ and $\mathrm{H}(\mathrm{f})_{2}$, the functional $\Psi$, introduced in (2.16), is well-defined, of class $C^{1}$, and weakly sequentially continuous, with

$$
\left\langle\Psi^{\prime}(u), v\right\rangle=\int_{\Omega} f^{*}(x, u) v d x
$$

for all $u, v \in W_{0}^{1, p}(\Omega)$. Moreover, the operator $\Psi^{\prime}: W_{0}^{1, p}(\Omega) \rightarrow W^{-1, p^{\prime}}(\Omega)$ is strongly continuous (i.e., $u_{n} \rightarrow u$ implies $\Psi^{\prime}\left(u_{n}\right) \rightarrow \Psi^{\prime}(u)$ ). 
Proof. From (2.10), (2.6) and Young's inequality, we have

$$
\begin{aligned}
|F(x, s)| & \leq \int_{0}^{|s|} f^{*}(x, t) d t \leq b_{1} \int_{0}^{s_{0}} \max \{t, \underline{u}(x)\}^{-\gamma} d t+\frac{b_{2}}{q}|s|^{q}+a(x)|s| \\
& =b_{1}\left(\int_{0}^{\underline{u}(x)} \underline{u}(x)^{-\gamma} d t+\int_{\underline{u}(x)}^{s_{0}} t^{-\gamma} d t\right)+\frac{b_{2}}{q}|s|^{q}+a(x)|s| \\
& \leq\left(1+\frac{1}{1-\gamma}\right) b_{1} s_{0}^{1-\gamma}+\frac{1}{q^{\prime}} a(x)^{q^{\prime}}+\frac{b_{2}+1}{q}|s|^{q},
\end{aligned}
$$

for all $x \in \Omega$ and $s \in \mathbb{R}$. The last member of (2.18) for $s=u^{+}(x)$ is a $L^{1}(\Omega)$ function. Thus, $\Psi$ is well-defined.

In order to prove the regularity of $\Psi$, let us compute its Gâteaux derivative. Take any $v \in$ $W_{0}^{1, p}(\Omega)$.

$$
\lim _{t \rightarrow 0^{+}} \frac{\Psi(u+t v)-\Psi(u)}{t}=\lim _{t \rightarrow 0^{+}} \int_{\Omega} \frac{F(x,(u+t v))-F(x, u)}{t} d x .
$$

Fix any $t>0$. According to Torricelli's theorem, one has

$$
\begin{aligned}
F(x,(u+t v))-F(x, u) & =t v\left(\int_{0}^{1}\left(\frac{d}{d s} F(x, u+s t v)\right) d s\right) \\
& =t v\left(\int_{0}^{1} f^{*}(x, u+s t v) d s\right)
\end{aligned}
$$

On the other hand, (2.17) furnishes

$$
f^{*}(x, y+z) \leq c_{1} d(x)^{-\gamma}+a(x)+b_{2} 2^{q-1}\left(|y|^{q-1}+|z|^{q-1}\right),
$$

for every $x \in \Omega$ and $y, z \in \mathbb{R}$. Plugging (2.20) into (2.19), and using Fubini's theorem, inequality (2.4), estimate (2.21), Lebesgue's dominated convergence theorem (argue as in (2.11)), we have

$$
\begin{aligned}
\left\langle\Psi^{\prime}(u), v\right\rangle= & \lim _{t \rightarrow 0^{+}} \frac{\Psi(u+t v)-\Psi(u)}{t} \\
& =\lim _{t \rightarrow 0^{+}} \int_{\Omega}\left(\int_{0}^{1} v f^{*}(x, u+s t v) d s\right) d x \\
& =\lim _{t \rightarrow 0^{+}} \int_{0}^{1}\left(\int_{\Omega} v f^{*}(x, u+s t v) d x\right) d s \\
& =\int_{0}^{1}\left(\int_{\Omega} v \lim _{t \rightarrow 0^{+}}\left(f^{*}(x, u+s t v)\right) d x\right) d s \\
& =\int_{\Omega} f^{*}(x, u) v d x
\end{aligned}
$$

for all $u, v \in W_{0}^{1, p}(\Omega)$.

First of all, observe that, for any $u \in W_{0}^{1, p}(\Omega)$ and $\left\{u_{n}\right\} \subseteq W_{0}^{1, p}(\Omega)$ such that $u_{n} \rightarrow u$ in $W_{0}^{1, p}(\Omega)$, the compactness of the embedding $W_{0}^{1, p}(\Omega) \hookrightarrow L^{q}(\Omega)$ for $q \in\left[1, p^{*}\right)$ implies that there exists $w \in L^{q}(\Omega)$ such that

$$
u_{n} \rightarrow u \text { in } L^{q}(\Omega), u_{n} \rightarrow u \text { a.e. in } \Omega \text { and }\left|u_{n}\right| \leq w
$$


for every $n \in \mathbb{N}$ (see [3, Theorem 4.9]). Now we prove that $\Psi^{\prime}: W_{0}^{1, p}(\Omega) \rightarrow W^{-1, p^{\prime}}(\Omega)$ is a continuous operator. To this aim, let $u_{n} \rightarrow u$ in $W_{0}^{1, p}(\Omega)$. According to (2.17), we have

$$
f^{*}\left(x, u_{n}\right) \leq c_{1} d(x)^{-\gamma}+a(x)+b_{2}|w|^{q-1}
$$

for all $x \in \Omega$ and $n \in \mathbb{N}$. Therefore, as above, Lebesgue's dominated convergence theorem and the continuity of $f^{*}(x, \cdot)$ for a.a. $x \in \Omega$ ensure that

$$
\lim _{n \rightarrow \infty}\left\langle\Psi^{\prime}\left(u_{n}\right), v\right\rangle=\int_{\Omega} \lim _{n \rightarrow \infty} f^{*}\left(x, u_{n}\right) v d x=\int_{\Omega} f^{*}(x, u) v d x=\left\langle\Psi^{\prime}(u), v\right\rangle .
$$

We conclude that $\Psi$ is of class $C^{1}$. In order to prove that $\Psi^{\prime}$ is strongly continuous, we let $u_{n} \rightarrow u$ in $W_{0}^{1, p}(\Omega)$. Observe that

$$
\lim _{n \rightarrow \infty}\left|\left\langle\Psi^{\prime}\left(u_{n}\right)-\Psi^{\prime}(u), v\right\rangle\right| \leq \lim _{n \rightarrow \infty} \int_{\Omega}\left|f^{*}\left(x, u_{n}\right)-f^{*}(x, u)\right||v| d x
$$

for any $v \in W_{0}^{1, p}(\Omega)$. Owing to (2.23)-(2.24), Lebesgue's dominated convergence theorem applies. Thus, (2.25) gives $\Psi^{\prime}\left(u_{n}\right) \rightarrow \Psi^{\prime}(u)$, as desired. The weak sequential continuity of $\Psi$ is a direct consequence of [33, Corollary 41.9].

Remark 2.1. We explicitly observe that the auxiliary function $f^{*}$ in (2.9) involves only a truncation from below of the reaction term $f$. Of course, it clearly appears that $f^{*}$ allows us to avoid the singularity of the function $f$.

However, to simplify the estimate of the parameter $\lambda^{*}$ defined in Theorem 3.1 , and at the same time to preserve the $C^{1}$-regularity property of the functional $\Psi$ given in (2.16), we needed to extend $f^{*}$ by evenness for $s<0$, because we cannot assume $f^{*}(x, s)=0$ for $s<0$.

On the other hand, since we do not make any truncation of $f$ from above by employing an appropriate supersolution of problem $\left(P_{\lambda, f}\right)$, we take advantage from that by using variational methods to obtain a first solution without assuming any asymptotic condition at infinity on $f$.

Remark 2.2. Estimate (2.18) implies that, for any $R>0$ and $u \in W_{0}^{1, p}(\Omega)$, there exists a positive constant $\Pi(R)$ such that

$$
\int_{\Omega(|u| \leq R)}|F(x, u(x))| d x \leq \Pi(R),
$$

where $\Omega(|u| \leq R)=\{x \in \Omega:-R \leq u(x) \leq R\}$. Analogously, estimates (2.10) and (2.6) ensure that, for any $R>0$ and $u \in W_{0}^{1, p}(\Omega)$, there exists a positive constant $\Pi^{\prime}(R)$

$$
\int_{\Omega(|u| \leq R)} f^{*}(x, u(x))|u(x)| d x \leq \Pi^{\prime}(R) .
$$

Variational methods are the essential tool to obtain our main results. In this connection, we apply the following general local minimum theorem to get a first solution for problem $\left(P_{\lambda, f}\right)$.

Theorem 2.1. Let $X$ be a reflexive Banach space, $\Phi: X \rightarrow \mathbb{R}$ and $\Psi: X \rightarrow \mathbb{R}$ two continuously Gâteaux differentiable functionals such that $\Phi$ is coercive and sequentially weakly lower semicontinuous, while $\Psi$ is sequentially weakly upper semicontinuous with $\inf _{X} \Phi=\Phi(0)=\Psi(0)$, and $r>0$. Then, for every

$$
\lambda \in] 0, \frac{r}{\sup _{\Phi^{-1}([0, r])} \Psi}[
$$


the functional $J_{\lambda}:=\Phi-\lambda \Psi$ has a critical point $u_{\lambda} \in \Phi^{-1}([0, r])$ satisfying $J_{\lambda}\left(u_{\lambda}\right) \leq J_{\lambda}(v)$ for any $v \in \Phi^{-1}([0, r])$.

Proof. The regularity assumptions on the functionals $\Phi$ and $\Psi$ imply that both $\Phi^{-1}([0, r])$ is weakly compact in $X$ and the functional $J_{\lambda}$ is sequentially weakly lower semicontinuous in $\Phi^{-1}([0, r])$. Therefore, Tonelli-Weierstrass's Theorem guarantees that there exists $u^{*} \in$ $\Phi^{-1}([0, r])$ such that

$$
J_{\lambda}\left(u^{*}\right)=\min _{\Phi^{-1}([0, r])} J_{\lambda}
$$

Hence, our conclusion follows arguing as in the proof of [7, Theorem 3.3].

Remark 2.3. A different proof of Theorem 2.1, based on non-smooth critical point theory, can be obtained, for instance, arguing as in [6, Remark 3.3]. For a general overview about this topic, we refer to [5].

In order to get multiplicity results, we will make use of the Mountain Pass Theorem by Ambrosetti and Rabinowitz [2]. We premise one of its main ingredients, the so-called PalaisSmale condition.

Definition 2.1 ((PS)). Let $X$ be a Banach space and $J \in C^{1}(X)$. We say that $J$ satisfies the PalaisSmale condition if any sequence $\left\{x_{n}\right\} \subseteq X$ such that $\left\{J\left(x_{n}\right)\right\}$ is bounded and $\left\|J^{\prime}\left(x_{n}\right)\right\|_{X^{*}} \rightarrow 0$ admits a convergent subsequence.

Theorem 2.2. Suppose that $X$ is a Banach space, and $J \in C^{1}(X)$ satisfies (PS)-condition. Let $x_{0}, x_{1} \in X$, and $\rho>0$ such that

$$
\max \left\{J\left(x_{0}\right), J\left(x_{1}\right)\right\} \leq \inf _{\partial B\left(x_{0}, \rho\right)} J=: \eta_{\rho}, \quad\left\|x_{1}-x_{0}\right\|_{X}>\rho .
$$

Set

$$
\Gamma:=\left\{\gamma \in C^{0}([0,1] ; X): \gamma(0)=x_{0}, \gamma(1)=x_{1}\right\}, \quad c:=\inf _{\gamma \in \Gamma} \sup _{t \in[0,1]} J(\gamma(t)) .
$$

Then $c \geq \eta_{\rho}$ and there exists $x \in X$ such that $J(x)=c$ and $J^{\prime}(x)=0$. Moreover, if $c=\eta_{\rho}$, then $x$ can be taken on $\partial B\left(x_{0}, \rho\right)$.

\section{MAin RESUlTS}

The following three positive constants $A, B$, and $r_{\lambda}^{*}$ play a crucial role in our existence result:

$$
\begin{gathered}
A=\left(1+\frac{1}{1-\gamma}\right) b_{1} s_{0}^{1-\gamma}|\Omega|+\frac{1}{q^{\prime}}\|a\|_{q^{\prime}}^{q^{\prime}} \\
B=\left(\frac{b_{2}+1}{q}\right) S_{q}^{q} p^{\frac{q}{p}},
\end{gathered}
$$

with $S_{q}$ arising from (2.2), and

$$
r_{\lambda}^{*} \begin{cases}>r: \lambda B r^{\frac{q}{p}}-r+\lambda A=0, & \text { if } 1<q<p, \\ >\frac{\lambda A}{1-\lambda B}, & \text { if } q=p, \\ =\left(\frac{A}{B} \frac{p}{q-p}\right)^{\frac{p}{q}}, & \text { if } p<q<p^{*} .\end{cases}
$$

Our first main result is the following. 
Theorem 3.1. Suppose that $\mathrm{H}(\mathrm{f})_{1}$ and $\mathrm{H}(\mathrm{f})_{2}$ hold. Set

$$
\lambda^{*}= \begin{cases}+\infty, & \text { if } 1<q<p, \\ \frac{1}{B}, & \text { if } q=p, \\ \frac{1}{q}\left(\frac{q-p}{A}\right)^{1-\frac{p}{q}}\left(\frac{p}{B}\right)^{\frac{p}{q}}, & \text { if } p<q<p^{*},\end{cases}
$$

where $A, B$, and $r_{\lambda}^{*}$ are defined in (3.1)-(3.2)-(3.3). Then, for any $\lambda \in\left(0, \lambda^{*}\right)$, there exists a weak solution $u^{*}=u^{*}(\lambda)$ to $\left(P_{\lambda, f}\right)$ with $\left\|u^{*}\right\| \leq\left(p r_{\lambda}^{*}\right)^{\frac{1}{p}}$.

Proof. Taking into account Lemma 2.2, our goal is to apply Theorem 2.1 to the energy functional $J_{\lambda}=\Phi-\lambda \Psi$ associated to problem $\left(P_{\lambda, f^{*}}\right)$, where

$$
\Phi: W_{0}^{1, p}(\Omega) \rightarrow \mathbb{R}, \quad \Phi(u)=\frac{1}{p}\|u\|^{p}
$$

and

$$
\Psi: W_{0}^{1, p}(\Omega) \rightarrow \mathbb{R}, \quad \Psi(u)=\int_{\Omega} F(x, u) d x,
$$

with $f^{*}$ and $F$ being defined in (2.9) and (2.15), respectively. Bearing in mind both Lemma 2.3 and the well-known properties of the $p$-Laplacian operator, in particular

$$
\left\langle\Phi^{\prime}(u), v\right\rangle=\int_{\Omega}|\nabla u|^{p-2} \nabla u \nabla v d x
$$

for all $u, v \in W_{0}^{1, p}(\Omega)$, we find that functionals $\Phi$ and $\Psi$ satisfy all the assumptions of Theorem 2.1, as well as that the critical points of $J_{\lambda}$ are weak solutions of problem $\left(P_{\lambda, f^{*}}\right)$.

Fix $r>0$. Owing to (2.18), (3.1), and (3.2), one has

$$
\frac{1}{r} \sup _{\|u\| \leq(p r)^{\frac{1}{p}}} \Psi(u) \leq \frac{A}{r}+B r^{\frac{q}{p}-1}=: \frac{1}{h(r)} .
$$

Notice that

$$
\lim _{r \rightarrow 0^{+}} h(r)=0
$$

for any $q \in\left(1, p^{*}\right)$,

$$
\lim _{r \rightarrow+\infty} h(r)= \begin{cases}+\infty, & \text { if } 1<q<p \\ \frac{1}{B}, & \text { if } q=p \\ 0, & \text { if } p<q<p^{*}\end{cases}
$$

and $h$ is strictly increasing whenever $q \in(1, p]$. If $p<q<p^{*}$, then a direct computation of the (unique) critical point of $h$ shows that

$$
\sup _{r>0} h(r)=h\left(\left(\frac{A}{B} \frac{p}{q-p}\right)^{\frac{p}{q}}\right)=\frac{1}{q}\left(\frac{q-p}{A}\right)^{1-\frac{p}{q}}\left(\frac{p}{B}\right)^{\frac{p}{q}} .
$$

Thus

$$
\sup _{r>0} h(r)=\lambda^{*} \leq \frac{r_{\lambda}^{*}}{\sup _{\Phi^{-1}\left(\left[0, r_{\lambda}^{*}\right]\right)} \Psi}
$$

where $r_{\lambda}^{*}$ is as in (3.3). Finally, we fix $\lambda \in\left(0, \lambda^{*}\right)$. The conclusion follows from Theorem 2.1, once we take $r=r_{\lambda}^{*}$. 
Remark 3.1. It is worth noticing that the constants $A$ and $B$ are related to both the estimate (2.18) and the geometry of the problem $\left(P_{\lambda, f}\right)$ (see also (2.2)). On the other hand, $r_{\lambda}^{*}$ gives the smallest radius of the ball in $W_{0}^{1, p}(\Omega)$ where a local minimum for the energy functional $J_{\lambda}$, associated to problem $\left(P_{\lambda, f^{*}}\right)$, is located. Finally, we point out that in the case $q \leq p$ the functional $J_{\lambda}$ turns out to be coercive.

Remark 3.2. The condition

(j) $\limsup _{s \rightarrow 0^{+}} f(x, s) s^{\gamma} \leq b_{1}$, uniformly w.r.t. $x \in \Omega$,

clearly means that there exists $\rho>0$ such that $f(x, s) s^{\gamma} \leq b_{1}$ for every $0<s<\rho$. If, in addition, we have that

(jj) the function

$$
M_{\rho}: \Omega \rightarrow[0, \infty), \quad M_{\rho}(x)=\sup _{s \in\left[\rho, s_{0}\right]} f(x, s),
$$

belongs to $L^{q^{\prime}}(\Omega)$,

then it follows that $\mathrm{H}(\mathrm{f})_{2}$ (ii) continues to hold whenever the function $a$ is replaced with the function $M_{\rho}+a \in L^{q^{\prime}}(\Omega)$.

Of course, $(\mathrm{jj})$ is guaranteed in the autonomous case, i.e., when $f$ does not depend on $x$.

Example 3.1. Let $h \in L^{\infty}(\Omega)$, essinf $h>0, \gamma \in(0,1), g_{1}: \Omega \times \mathbb{R} \rightarrow[0,+\infty)$ a Carathéodory function such that

$$
g_{1}(x, s) \leq c_{1}+c_{2} s^{q-1}
$$

for all $x \in \Omega$ and $s \geq 0$, with $c_{1}, c_{2} \geq 0$ and $q>1$. Let $g_{2}: \mathbb{R} \rightarrow[0,+\infty)$ be a continuous function such that

$$
\limsup _{s \rightarrow 0^{+}} \frac{g_{2}(s)}{s^{\gamma}}=+\infty, \quad \limsup _{s \rightarrow 0^{+}} g_{2}(s) \leq b_{1}, \quad \limsup _{s \rightarrow+\infty} \frac{g_{2}(s)}{s^{q-1+\gamma}}<+\infty
$$

for some $b_{1} \geq 0$ and $q \in\left(1, p^{*}\right)$.

Nonlinearities that satisfy hypotheses $\mathrm{H}(\mathrm{f})_{1}-\mathrm{H}(\mathrm{f})_{2}$ are, e.g.,

$$
f(x, s)=\frac{h(x)}{s^{\gamma}}+g_{1}(x, s),
$$

and

$$
f(x, s)=\frac{g_{2}(s)}{s^{\gamma}} .
$$

Below, we expose our main result concerning the existence of two weak solutions for problem $\left(P_{\lambda, f}\right)$.

Lemma 3.1. Suppose that $\mathrm{H}(\mathrm{f})_{1}, \mathrm{H}(\mathrm{f})_{2}$, and $\left(A R^{+}\right)$hold. Then the functional $J_{\lambda}$ satisfies (PS) and is unbounded from below.

Proof. This proof is patterned after [16, Theorems 15 and 16]. Let us consider a sequence $\left\{u_{n}\right\} \subseteq W_{0}^{1, p}(\Omega)$ such that $\left\{J_{\lambda}\left(u_{n}\right)\right\}$ is bounded and $\left\{J_{\lambda}^{\prime}\left(u_{n}\right)\right\}$ converges to zero. In other words, for any $n \in \mathbb{N}$,

$$
\frac{1}{p}\left\|u_{n}\right\|^{p}-\lambda \int_{\Omega} F\left(x, u_{n}^{+}\right) d x \leq c_{1}
$$

and

$$
\left.\left|\int_{\Omega}\right| \nabla u_{n}\right|^{p-2} \nabla u_{n} \nabla v d x-\lambda \int_{\Omega} f^{*}\left(x, u_{n}\right) v d x \mid \leq\|v\| \quad \forall v \in W_{0}^{1, p}(\Omega)
$$


hold true for an appropriate constant $c_{1}>0$, independent of $n$. We will prove that $\left\{u_{n}\right\}$ is bounded by showing the property for $\left\{u_{n}^{-}\right\}$and $\left\{u_{n}^{+}\right\}$. Exploiting (3.9) with $v=-u_{n}^{-}$leads to

$$
\left\|u_{n}^{-}\right\|^{p} \leq\left\|u_{n}^{-}\right\|^{p}+\lambda \int_{\Omega} f\left(x, u_{n}\right) u_{n}^{-} d x \leq\left\|u_{n}^{-}\right\|
$$

and hence $\left\|u_{n}^{-}\right\| \leq 1$ for all $n \in \mathbb{N}$. Let $\Omega_{n}:=\left\{x \in \Omega: u_{n}^{+}(x) \geq R\right\}$ and $\Omega_{n}^{\prime}:=\Omega \backslash \Omega_{n}$ for any $n \in \mathbb{N}$. According to (2.26), one has

$$
\int_{\Omega_{n}^{\prime}} F\left(x, u_{n}^{+}\right) d x \leq \Pi(R)
$$

while $\left(A R^{+}\right)$gives

$$
\begin{aligned}
\int_{\Omega_{n}} F\left(x, u_{n}^{+}\right) d x & =\int_{\Omega_{n}}\left(F\left(x, s_{1}\right)+\int_{s_{1}}^{u_{n}^{+}} f(x, t) d t\right) d x \\
& \leq \Pi\left(s_{1}\right)+\frac{1}{\mu} \int_{\Omega_{n}} f\left(x, u_{n}^{+}\right) u_{n}^{+} d x \\
& \leq \Pi(R)+\frac{1}{\mu} \int_{\Omega} f^{*}\left(x, u_{n}\right) u_{n}^{+} d x-\frac{1}{\mu} \int_{\Omega_{n}^{\prime}} f^{*}\left(x, u_{n}\right) u_{n}^{+} d x
\end{aligned}
$$

On the other hand, from (2.27), we get

$$
\left|\int_{\Omega_{n}^{\prime}} f^{*}\left(x, u_{n}\right) u_{n}^{+} d x\right| \leq \Pi^{\prime}(R) .
$$

Then, (3.11) becomes

$$
\int_{\Omega_{n}} F\left(x, u_{n}^{+}\right) d x \leq \Pi(R)+\frac{1}{\mu} \int_{\Omega} f^{*}\left(x, u_{n}\right) u_{n}^{+} d x+\frac{1}{\mu} \Pi^{\prime}(R) .
$$

From (3.8), we infer

$$
\begin{aligned}
& \frac{1}{p}\left\|u_{n}^{+}\right\|^{p} \leq \frac{1}{p}\left\|u_{n}\right\|^{p} \leq c_{1}+\lambda\left(\int_{\Omega_{n}} F\left(x, u_{n}^{+}\right) d x+\int_{\Omega_{n}^{\prime}} F\left(x, u_{n}^{+}\right) d x\right) \\
& \leq c_{1}+\lambda\left(2 \Pi(R)+\frac{1}{\mu} \int_{\Omega} f^{*}\left(x, u_{n}\right) u_{n}^{+} d x+\frac{1}{\mu} \Pi^{\prime}(R)\right) \\
& =: \frac{\lambda}{\mu} \int_{\Omega} f^{*}\left(x, u_{n}\right) u_{n}^{+} d x+c_{2},
\end{aligned}
$$

while (3.9), tested with $v=u_{n}^{+}$, yields

$$
-\frac{1}{\mu}\left\|u_{n}^{+}\right\|^{p}-\frac{1}{\mu}\left\|u_{n}^{+}\right\| \leq-\frac{\lambda}{\mu} \int_{\Omega} f^{*}\left(x, u_{n}\right) u_{n}^{+} d x .
$$

Adding term by term (3.14) and (3.15), we conclude

$$
\left(\frac{1}{p}-\frac{1}{\mu}\right)\left\|u_{n}^{+}\right\|^{p}-\frac{1}{\mu}\left\|u_{n}^{+}\right\| \leq c_{2} .
$$

Hence, $\left\{u_{n}^{+}\right\}$is bounded in $W_{0}^{1, p}(\Omega)$. Up to subsequences, we have $u_{n} \rightarrow u$ in $W_{0}^{1, p}(\Omega)$. Since $\Psi^{\prime}$ is strongly continuous (see Lemma 2.16), by (3.9) we have

$$
\lim _{n \rightarrow \infty}\left\langle\Phi^{\prime}\left(u_{n}\right), u_{n}-u\right\rangle=\lim _{n \rightarrow \infty}\left\langle J_{\lambda}^{\prime}\left(u_{n}\right), u_{n}-u\right\rangle+\lambda \lim _{n \rightarrow \infty}\left\langle\Psi^{\prime}\left(u_{n}\right), u_{n}-u\right\rangle=0 .
$$


Property $\left(\mathrm{S}_{+}\right)$of the $p$-Laplacian operator (see [25, Proposition 2.72]) forces $u_{n} \rightarrow u$ in $W_{0}^{1, p}(\Omega)$. In conclusion, $J_{\lambda}$ satisfies (PS).

Next, we prove that $J_{\lambda}$ is unbounded from below. For all $M \geq 1$, set $\Omega_{M}=\left\{x \in \Omega: M \varphi_{p}(x) \geq\right.$ $R\}$ and $\Omega_{M}^{\prime}:=\Omega \backslash \Omega_{M}$, with $\varphi_{p}$ as in (2.3). Fix $\bar{M}>0$ such that $\left|\Omega_{\bar{M}}\right|>0$ (this choice is possible because $\Omega_{M} \uparrow \Omega$ for $\left.M \rightarrow+\infty\right)$. Obviously, $\Omega_{\bar{M}} \subseteq \Omega_{M}$ for all $M \geq \bar{M}$, and hence $\left|\Omega_{M}\right|>0$. From $\left(A R^{+}\right)$, we have, for any $\sigma \geq R$,

$$
\frac{\mu}{\sigma} \leq \frac{f(x, \sigma)}{\int_{s_{1}}^{\sigma} f(x, t) d t}=\frac{\frac{\partial}{\partial \sigma}\left(\int_{s_{1}}^{\sigma} f(x, t) d t\right)}{\int_{s_{1}}^{\sigma} f(x, t) d t}
$$

Let $s \geq R$. Integrating (3.16) in $\sigma$ on $[R, s]$, we get

$$
\log \left[\left(\frac{s}{R}\right)^{\mu}\right] \leq \log \left(\frac{\int_{s_{1}}^{s} f(x, t) d t}{\int_{s_{1}}^{R} f(x, t) d t}\right) .
$$

Hence,

$$
\int_{s_{1}}^{s} f(x, t) d t \geq\left(R^{-\mu} \int_{s_{1}}^{R} f(x, t) d t\right) s^{\mu}=: G(x) s^{\mu} \quad \forall s \geq R
$$

with $G(x)=R^{-\mu} \int_{s_{1}}^{R} f(x, t) d t>0$ for all $x \in \Omega$, by virtue of $\left(A R^{+}\right)$. Moreover, $\mathrm{H}(\mathrm{f})_{2}(\mathrm{ii})$ ensures that $G \in L^{1}(\Omega)$. We claim that $\lim _{M \rightarrow+\infty} J_{\lambda}\left(M \varphi_{p}\right)=-\infty$. To this end, we observe that

$$
\begin{aligned}
\int_{\Omega_{M}} F\left(x, M \varphi_{p}\right) d x & =\int_{\Omega_{M}}\left(F\left(x, s_{1}\right)+\int_{s_{1}}^{M \varphi_{p}} f(x, t) d t\right) d x \\
& \geq-\Pi\left(s_{1}\right)+M^{\mu} \int_{\Omega_{M}} G(x) \varphi_{p}^{\mu} d x \\
& \geq M^{\mu}\left(\frac{R}{\bar{M}}\right)^{\mu} \int_{\Omega_{\bar{M}}} G(x) d x-\Pi(R)
\end{aligned}
$$

for $M \geq \bar{M}$. Notice that $\int_{\Omega_{\bar{M}}} G(x) d x>0$, because $G>0$ in $\Omega$ and $\left|\Omega_{\bar{M}}\right|>0$. On the other hand, (2.26) gives

$$
\left|\int_{\Omega_{M}^{\prime}} F\left(x, M \varphi_{p}\right) d x\right| \leq \Pi(R) .
$$

Plugging (3.18)-(3.19) together, we find

$$
\begin{aligned}
J_{\lambda}\left(M \varphi_{p}\right) & =\frac{1}{p}\left\|M \varphi_{p}\right\|^{p}-\lambda\left(\int_{\Omega_{M}} F\left(x, M \varphi_{p}\right) d x+\int_{\Omega_{M}^{\prime}} F\left(x, M \varphi_{p}\right) d x\right) \\
& \leq \frac{\lambda_{1}}{p} M^{p}-\lambda\left(\frac{R}{\bar{M}}\right)^{\mu} M^{\mu} \int_{\Omega_{\bar{M}}} G(x) d x+2 \lambda \Pi(R) \rightarrow-\infty
\end{aligned}
$$

for $M \rightarrow+\infty$. The claim is proved.

Theorem 3.2. Suppose that $\mathrm{H}(\mathrm{f})_{1}$ and $\mathrm{H}(\mathrm{f})_{2}$ hold. Let $f$ be satisfying $\left(A R^{+}\right)$. Then, for any $\lambda \in\left(0, \lambda^{*}\right)$, problem $\left(P_{\lambda, f}\right)$ admits at least two weak solutions $u^{*}(\lambda)$ and $\tilde{u}(\lambda)$, where $\lambda^{*}$ is defined in (3.4).

Proof. Fix $\lambda \in\left(0, \lambda^{*}\right)$ and consider $r_{\lambda}^{*}$, defined by (3.3). Set $r^{\prime}:=\left(p r_{\lambda}^{*}\right)^{\frac{1}{p}}$, and

$$
B\left(0, r^{\prime}\right):=\left\{u \in W_{0}^{1, p}(\Omega):\|u\|<r^{\prime}\right\} .
$$


The existence of a solution $u^{*} \in \bar{B}\left(0, r^{\prime}\right)$ is guaranteed by Theorem 3.1, and $u^{*}$ is a minimizer of the restriction to $\bar{B}\left(0, r^{\prime}\right)$ of the functional $J_{\lambda}$. Without loss of generality, we can suppose $u^{*}$ to be a proper minimizer, i.e.,

$$
J_{\lambda}\left(u^{*}\right)<J_{\lambda}(u) \quad \forall u \in \bar{B}\left(0, r^{\prime}\right) \backslash\left\{u^{*}\right\} .
$$

According to Lemma 3.1, the functional $J_{\lambda}$ satisfies (PS) and is unbounded from below. Then, for any $M>0$ sufficiently large, (3.20)-(3.21) imply

$$
J_{\lambda}\left(M \varphi_{p}\right)<J_{\lambda}\left(u^{*}\right) \leq \inf _{\partial B\left(0, r^{\prime}\right)} J_{\lambda} .
$$

Taking a larger $M$ if necessary, we can also suppose

$$
\left\|M \varphi_{p}-u^{*}\right\|>r^{\prime}
$$

Owing to (3.22)-(3.23), Theorem 2.2 ensures the existence of $\tilde{u} \in W_{0}^{1, p}(\Omega)$ such that $J_{\lambda}(\tilde{u})>$ $J_{\lambda}\left(u^{*}\right)$ (by (3.21)) and $J_{\lambda}^{\prime}(\tilde{u})=0$ (so $\tilde{u}$ is a solution to $\left.\left(P_{\lambda, f}\right)\right)$.

Remark 3.3. It is worth pointing out that all the above results remain true even for $q=1$ (cf. $\left.\mathrm{H}(\mathrm{f})_{2}\right)$. In this case, the function $a$ is assumed to be essentially bounded.

\section{Acknowledgments}

The paper was partially supported by PRIN 2017- Progetti di Ricerca di rilevante Interesse Nazionale, "Nonlinear Differential Problems via Variational, Topological and Set-valued Methods"(Grant No. 2017AYM8XW).

\section{REFERENCES}

[1] R.A. Adams, Sobolev spaces, Pure and Applied Mathematics 65, Academic Press, New York-London, 1975.

[2] A. Ambrosetti, P.H. Rabinowitz, Dual variational methods in critical point theory and applications, J. Funct. Anal. 14 (1973), 349-381.

[3] H. Brezis, Functional analysis, Sobolev spaces and partial differential equations, Universitext, Springer, New York, 2011.

[4] H. Brezis, L. Nirenberg, $H^{1}$ versus $C^{1}$ local minimizers, C.R.A.S. Paris 317 (1993), 465-472.

[5] G. Bonanno, A critical point theorem via the Ekeland variational principle, Nonlinear Anal. 75 (2012), 29923007.

[6] G. Bonanno, P. Candito, Non-differentiable functionals and applications to elliptic problems with discontinuous nonlinearities, J. Differential Equations 44 (2008), no. 12, 3031-3059.

[7] G. Bonanno, P. Candito, G. D’Aguì, Variational methods on finite dimensional Banach space and discrete problems, Ad. Nonlinear Stud. 14 (2014), no. 4, 915-939.

[8] G. Bonanno, G. D’ Aguì, Two non-zero solutions for elliptic Dirichlet problems, Z. Anal. Anwend. 35 (2016), no. 4, 449-464.

[9] F. Brock, L. Itturiaga, P. Ubilla, A multiplicity result for the p-Laplacian involving a parameter, Annales Inst. H. Poincaré Analyse Non Linéaire 9 (2008), 1371-1386.

[10] P. Candito, S. Carl, R. Livrea, Multiple solutions for quasilinear elliptic problems via critical points in open sublevels and truncation principles, J. Math. Anal. Appl. 395 (2012), no. 1, 156-163.

[11] P. Candito, S. Carl, R. Livrea, Critical points in open sublevels and multiple solutions for parameterdepending quasilinear elliptic equations, Adv. Differential Equations 19 (2014), no. 11-12, 1021-1042.

[12] A. Cianchi, V.G. Maz'ya, Quasilinear elliptic problems with general growth and merely integrable, or measure, data, Nonlinear Anal. 164 (2017), 189-215.

[13] G. M. Coclite, M.M. Coclite, On a Dirichlet problem in bounded domains with singular nonlinearity, Discrete Contin. Dyn. Syst. 33 (2013), no. 11-12, 4923-4944. 
[14] M. G. Crandall, P. H. Rabinowitz, L. Tartar, On a Dirichlet problem with a singular nonlinearity, Comm. Partial Differential Equations 2 (1977), no. 2, 193-222.

[15] R. Dhanya, E. Ko, R. Shivaji, A three solution theorem for singular nonlinear elliptic boundary value problems, J. Math. Anal. Appl. 424 (2015), no. 1, 598-612.

[16] G. Dinca, P. Jebelean, J. Mawhin, Variational and topological methods for Dirichlet problems with pLaplacian, Port. Math. (N.S.) 58 (2001), no. 3, 339-378.

[17] J.P. García Azorero, J.J. Manfredi, I. Peral Alonso, Sobolev versus Hölder local minimizers and global multiplicity for some quasilinear elliptic equations, Commun. Contemp. Math. 2 (2000), no. 3, 385-404.

[18] M. Ghergu, V.D. Rădulescu, Singular Elliptic Problems: Bifurcation and Asymptotic Analysis, Oxford Univ. Press, Oxford, 2008.

[19] J. Giacomoni, K. Saoudi, $W_{0}^{1, p}$ versus $C^{1}$ local minimizers for a singular and critical functional, J. Math. Anal. Appl. 363 (2010), no. 2, 697-710.

[20] J. Giacomoni, I. Schindler, P. Takac, Sobolev versus Hölder minimizers and global multiplicity for a singular and quasilinear equation, Annali Scuola Normale Superiore Pisa, Cl. Sci., 6:1 (2007), 117-158.

[21] J.V.A. Gonçalves, M.C. Rezende, C.A. Santos, Positive solutions for a mixed and singular quasilinear problem, Nonlinear Anal. 74 (2011), no. 1, 132-140.

[22] D.D. Hai, On a class of singular p-Laplacian boundary value problems, J. Math. Anal. Appl. 383 (2011), no. 2, 619-626.

[23] A.C. Lazer, P.J. Mckenna, On a singular nonlinear elliptic boundary value problem, Proc. Amer. Math. Soc. 111 (1991), no. 3, 721-730.

[24] G. Liebermann, The natural generalization of the natural conditions of Ladyzhenskaya and Ural'tseva for elliptic equations, Comm. Partial Differential Equations 16 (1991), 311-361.

[25] D. Motreanu, V.V. Motreanu, N.S. Papageorgiou, Topological and variational methods with applications to nonlinear boundary value problems, Springer, New York, 2014.

[26] N.S. Papageorgiou, G. Smyrlis, A bifurcation-type theorem for singular nonlinear elliptic equations, Methods Appl. Anal. 22 (2015), no. 2, 147-170.

[27] I. Peral, Multiplicity of Solutions for the p-Laplacian, ICTP Lecture Notes of the Second School of Nonlinear Functional Analysis and Applications to Differential Equations, Trieste, 1997.

[28] K. Perera, E.A.B. Silva, Existence and multiplicity of positive solutions for singular quasilinear problems, J. Math. Anal. Appl. 323 (2006), no. 2, 1238-1252.

[29] K. Perera, Z. Zhang, Multiple positive solutions of singular $p$-Laplacian problems by variational methods, Bound. Value Probl. 3 (2005), 377-382.

[30] P. Pucci, J. Serrin, The maximum principle, Prog. Nonlinear Differential Equations Appl. 73, Birkhäuser Verlag, Basel, 2007.

[31] C.A. Stuart, Existence and approximation of solutions of non-linear elliptic equations, Math. Z. 147 (1976), 53-63.

[32] G. Talenti, Best constants in Sobolev inequalities, Ann. Mat. Pura Appl. 110 (1976), 353-372.

[33] E. Zeidler, Nonlinear functional analysis and its applications III: Variational methods and optimization, Springer-Verlag, New York, 1985. 